\title{
Phosphorylation of histone H3 Ser10 establishes a hierarchy for subsequent intramolecular modification events
}

\author{
Stamatios Liokatis ${ }^{1}$, Alexandra Stützer ${ }^{2}$, Simon J Elsässer ${ }^{3,5}$, Francois-Xavier Theillet $^{1}$, Rebecca Klingberg ${ }^{4}$, \\ Barth van Rossum ${ }^{1}$, Dirk Schwarzer ${ }^{4,5}$, C David Allis ${ }^{3}$, Wolfgang Fischle ${ }^{2}$ \& Philipp Selenko $^{1}$ \\ Phosphorylation of Ser10 of histone $\mathrm{H} 3$ regulates chromosome condensation and transcriptional activity. Using time-resolved, \\ high-resolution NMR spectroscopy, we demonstrate that histone H3 Ser10 phosphorylation inhibits checkpoint kinase 1 \\ (Chk1)- and protein kinase C (PKC)-mediated modification of Thr11 and Thr6, the respective primary substrate sites of these \\ kinases. On unmodified $\mathrm{H} 3$, both enzymes also target Ser10 and thereby establish autoinhibitory feedback states on individual \\ H3 tails. Whereas phosphorylated Ser10 does not affect acetylation of Lys14 by Gcn5, phosphorylated Thr11 impedes acetylation. \\ Our observations reveal mechanistic hierarchies of $\mathrm{H} 3$ phosphorylation and acetylation events and provide a framework for \\ intramolecular modification cross-talk within the $\mathrm{N}$ terminus of histone $\mathrm{H} 3$.
}

\begin{abstract}
To better understand the range of cross-talk between post-translational histone modifications, we set out to explore the mechanistic hierarchies that govern histone $\mathrm{H} 3$ phosphorylation events ${ }^{1-4}$. We focused on protein kinases that modify four well-characterized substrate sites on the N terminus of histone H3: Thr6, Ser10, Thr11 and Ser28. Phosphorylation of these sites signals different biological outcomes. During interphase, Ser10 phosphorylation by mitogen- and stressactivated protein kinase (Msk1) marks transcriptional activation ${ }^{5}$, whereas Ser10 and Ser28 are phosphorylated by Aurora kinase B (AurB) at the onset of mitosis when chromosomes condense ${ }^{6}$. Phosphorylation of H3 Thr6 by PKC functions in androgen receptormediated gene expression by preventing demethylation of Lys4 (ref. 7). It also fine-tunes the specificities of chromatin-interaction domains for differentially methylated forms of Lys4 (ref. 8). Phosphorylation of Thr11 by PKC-related kinase 1 (PRK1) identifies active androgen receptor-dependent target genes (AREs) ${ }^{9}$, whereas modification of centromeric Thr11 by death-associated protein-like kinase (Dlk or ZIP1) signals the onset of mitosis ${ }^{10}$. In interphase cells, Thr11 phosphorylation by Chk1 is associated with gene activation, whereas loss of Thr11 phosphorylation upon DNA damage leads to transcriptional repression and reduced histone acetylation ${ }^{11}$.
\end{abstract}

\section{RESULTS}

Phosphorylation of $\mathrm{H} 3 \mathrm{Thr} 11$ and Ser10 by Chk1

To study phosphorylation by Chk1, we reacted uniformly ${ }^{15} \mathrm{~N}$-labeled histone $\mathrm{H} 3$ (residues 1-33) with the recombinant kinase and monitored phosphorylation of individual $\mathrm{H} 3$ residues by high-resolution NMR spectroscopy ${ }^{12}$. As previously reported, Chk1 phosphorylated H3 Thr11 (ref. 11). However, we also detected phosphorylation of Ser10 in approximately equal proportions (Fig. 1a). Time-resolved NMR monitoring of the Chk1 reaction revealed that phosphorylation of Ser10 and Thr11 was accomplished with comparable rates (Fig. 1b). Modification of Ser10 was slightly preferred over Thr11 phosphorylation, and this propensity was unaffected by changes in substrate concentration (Fig. 1c). Surprisingly, Chk1 never phosphorylated both sites on the same substrate molecule, so that Thr11 and Ser10 phosphorylation occurred in a mutually exclusive manner on separate $\mathrm{H} 3$ copies but never occurred together on the same substrate molecule. This phosphorylation behavior was confirmed by NMR analysis of synthetic $\mathrm{H} 3$ peptides phosphorylated at Ser10 or Thr11. (Fig. 1d). To investigate whether monospecific Ser10 and Thr11 phosphorylation by Chk1 also occurred in full-length or nucleosomal histone $\mathrm{H} 3$, we employed a set of antibodies that selectively recognized monophosphorylated H3 Ser10 and Thr11 substrates but not a doubly phosphorylated $\mathrm{H} 3$ peptide that contained both modifications on the same molecule (Fig. 1e). We then reacted full-length histone $\mathrm{H} 3$ as well as reconstituted mononucleosomes with Chk1 and probed for phosphoSer10 (pSer10) and phospho-Thr11 (pThr11) (Fig. 1f). Western blot detection indicated the presence of monophosphorylated Ser10 and Thr11 in both substrates. Although, using these antibodies, we could not rule out that doubly phosphorylated species were additionally present, we concluded that Chk1 displayed similar monospecific phosphorylation properties on full-length and nucleosomal histone $\mathrm{H} 3$.

\section{Nonreciprocal Ser10 phosphorylation hierarchies}

The mutually exclusive nature of Chk1's modification behavior suggested that the kinase did not engage in Thr11 modification when

\footnotetext{
${ }^{1}$ Department of NMR-supported Structural Biology, Leibniz Institute of Molecular Pharmacology (FMP Berlin), Berlin, Germany. ${ }^{2}$ Laboratory of Chromatin Biochemistry, Max Planck Institute for Biophysical Chemistry, Göttingen, Germany. ${ }^{3}$ Laboratory of Chromatin Biology and Epigenetics, Rockefeller University, New York, New York, USA. ${ }^{4}$ Department of Chemical Biology, Leibniz Institute of Molecular Pharmacology (FMP Berlin), Berlin, Germany. ${ }^{5}$ Present addresses: MRC Laboratory of Molecular Biology, Cambridge, UK (S.J.E.); Interfaculty Institute of Biochemistry (IFIB), University of Tübingen, Tübingen, Germany (D.S.). Correspondence should be addressed to P.S. (selenko@fmp-berlin.de).
} 
a
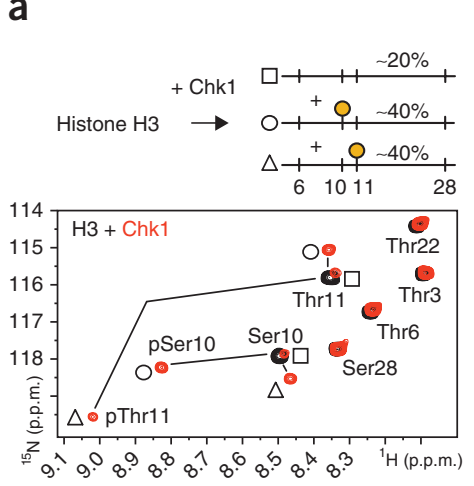

Figure 1 Mutually exclusive phosphorylation of H3 Thr11 and Ser10 by Chk1. (a) Superposition of $2 \mathrm{D}^{1} \mathrm{H}-{ }^{15} \mathrm{~N}$ NMR spectra (selected region) of uniform ${ }^{15} \mathrm{~N}$ isotope-labeled, unmodified recombinant histone $\mathrm{H} 3$ (residues

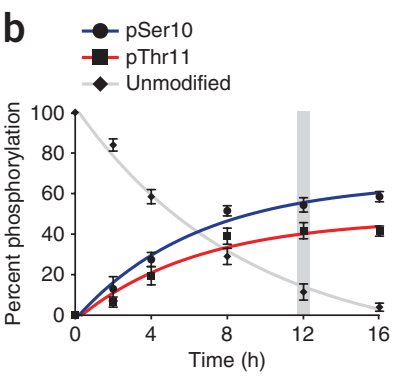

C

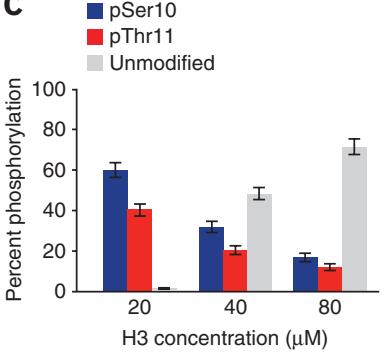

d

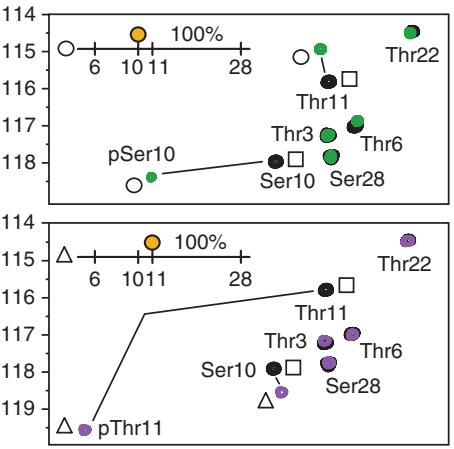

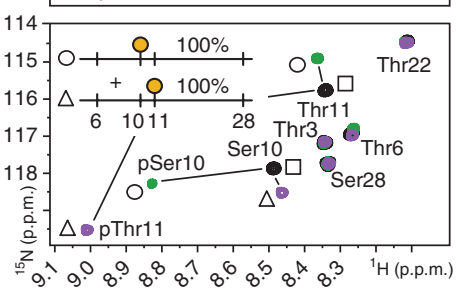

e

Concentration (pmol) 4010240102 H3 (1-33) H3 pThr3 H3 pThr6 H3 pSer10 H3 pThr11 H3 pSer10 and pThr11 H3 pSer28
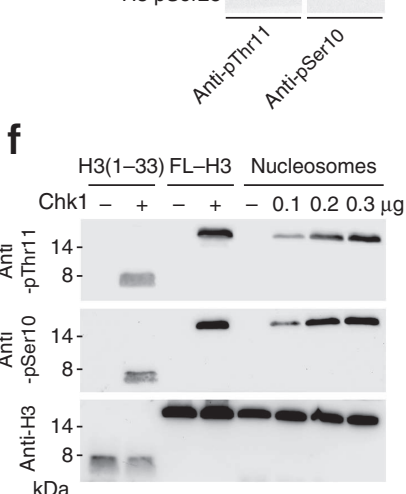

1-33) before (black) and after phosphorylation with Chk1 (red). (b) Time-resolved NMR profiling of Chk1 reactions via cross-peak integrations of pSer10 and NMR signals at the indicated time points from two independent experiments. Error bars represent s.d. The gray bar refers to the NMR spectrum shown in a. (c) pSer10 and pThr11 levels at Chk1 reaction endpoints (16 h) at different H3 substrate concentrations (two independent experiments). Error bars represent s.d. (d) Natural-abundance $2 \mathrm{D}^{1} \mathrm{H}_{-}{ }^{15} \mathrm{~N}$ NMR spectra (top and middle) and overlay of 2D NMR spectra (bottom). Black, residues 1-33 of unmodified synthetic histone H3; green, synthetic pSer10 H3; purple, synthetic pThr11. (e) Dot blot characterization of anti-pThr11 and anti-pSer10 antibodies on synthetic-H3 reference peptides. (f) Western blot analysis of Chk1 reactions with peptide H3, full-length (FL) H3 and reconstituted mononucleosomes.

Ser10 was already phosphorylated. To experimentally address this notion, we prephosphorylated uniformly ${ }^{15} \mathrm{~N}$-labeled histone $\mathrm{H} 3$ at Ser10 with either AurB ${ }^{13}$ or Msk1 (ref. 14). No additional phosphorylation of Thr11 by Chk1 was detected on these substrates, even after prolonged incubation with an excess of kinase (Supplementary Fig. 1a). To unambiguously determine the inhibitory potential of phosphorylated Ser10 on the Chk1-mediated modification of Thr11, we used $\left[{ }^{32} \mathrm{P}\right] \mathrm{ATP}$ incorporation assays. We reacted unmodified and
Ser10-phosphorylated H3 peptides (residues 1-15) with Chk1 and found no detectable levels of ${ }^{32} \mathrm{P}$ incorporation in the pSer10 substrates (Fig. 2a). To verify that this behavior was similarly preserved in a nucleosomal context, we prepared reconstituted mononucleosomes that we either incubated with AurB, to phosphorylate Ser10 and Ser28, or left unreacted (Supplementary Fig. 1b). Both samples were heat treated to inactivate AurB, and nucleosome integrity was confirmed by native PAGE DNA electrophoresis (Supplementary Fig. 1c). a

b

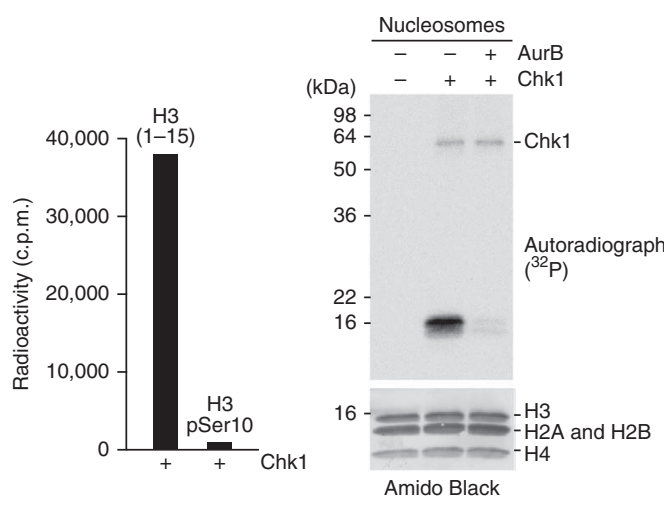

c
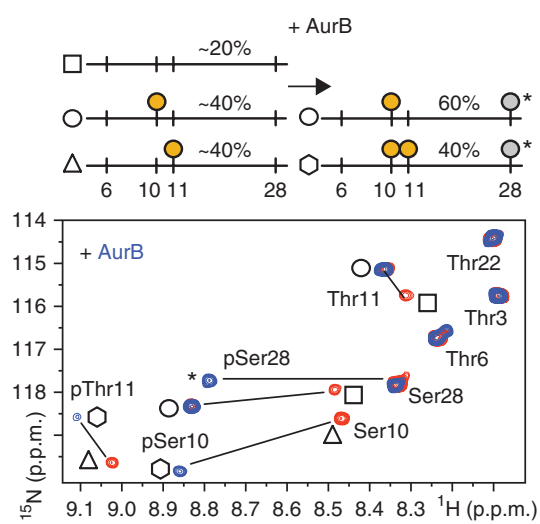

d

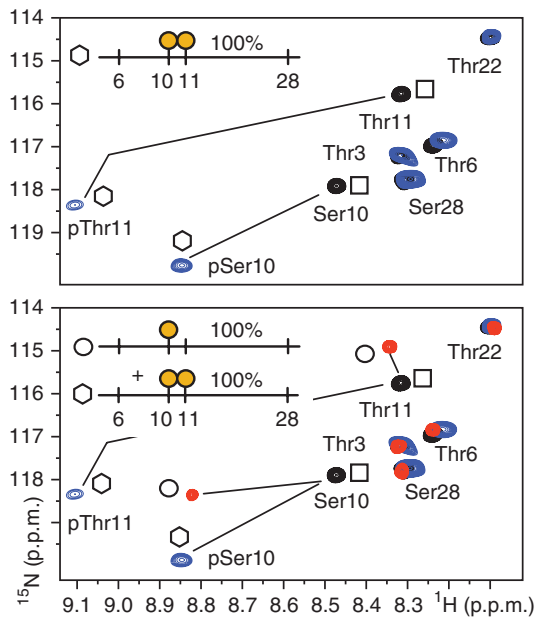

Figure 2 Nonreciprocal Ser10 phosphorylation hierarchies. (a) Chk1 [ ${ }^{32}$ P]ATP incorporation assay of synthetic unmodified and pSer10 H3 peptides. (b) Chk1 [ ${ }^{32} \mathrm{P}$ ]ATP incorporation assay of unmodified (lane 2) and H3 pSer10 and pSer28-modified nucleosomes (lane 3). (c) Overlay of 2D ${ }^{1} \mathrm{H}-{ }^{15} \mathrm{~N}$ NMR spectra of a mixture of uniformly ${ }^{15} \mathrm{~N}$ isotope-labeled histone $\mathrm{H} 3$ monophosphorylated at either Ser10 or Thr11 before (red) and after (blue) modification with AurB. Species doubly phosphorylated at Ser10 and Thr11 are generated by AurB. Onset of Ser28 phosphorylation is marked with an asterisk. (d) Natural-abundance 2D ${ }^{1} \mathrm{H}_{-}{ }^{15} \mathrm{~N}$ NMR spectra (top) and superposition of NMR spectra of synthetic H3 peptides (bottom). Black, unmodified synthetic H3; blue, synthetic peptide phosphorylated on both Ser10 and Thr11; red, pSer10. 
a

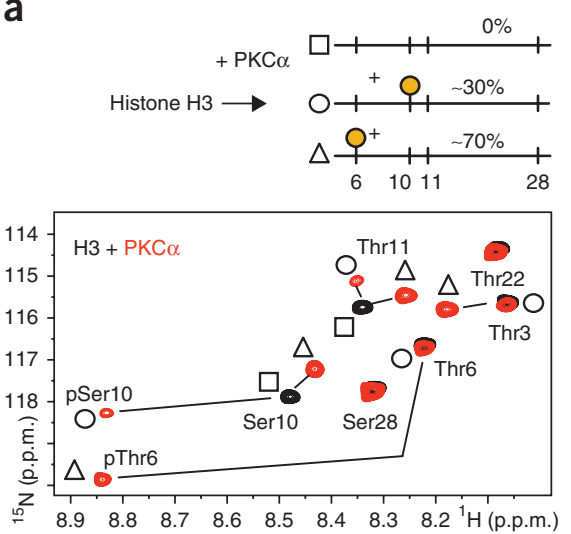

Figure 3 Mutually exclusive phosphorylation of H3 Thr6 (pThr6) and Ser10 by PKC $\alpha$. (a) Superposition of $2 \mathrm{D}^{1} \mathrm{H}-{ }^{15} \mathrm{~N}$ NMR spectra of ${ }^{15} \mathrm{~N}$ isotope-labeled histone $\mathrm{H} 3$ (black) and spectra upon phosphorylation with PKC $\alpha$ (red). (b) Time-resolved NMR profiling of $\mathrm{PKC} \alpha$ reactions via cross-peak integrations of pSer10 and pThr6 NMR signals at indicated time points from two independent experiments. Error bars represent s.d. The gray bar refers to the NMR spectrum in a. (c) pSer10 and pThr6 levels at PKC $\alpha$-reaction endpoints (16 h) at different $\mathrm{H} 3$ substrate

concentrations (two independent experiments).

Error bars represent s.d. (d) Natural-abundance

$2 \mathrm{D}^{1} \mathrm{H}^{15} \mathrm{~N}$ NMR spectra (top) of unmodified synthetic H3 (black) and synthetic pThr6 H3 (blue). Overlay of

2D NMR spectra (bottom) of unmodified (black) and monophosphorylated H3 Ser10 (green) and Thr6 peptides (blue). (e) Prephosphorylation of Thr6 and Ser10 by PKC $\alpha$ (red) does not prevent completion of Ser10 phosphorylation of pThr6 substrates by AurB (blue). Species doubly phosphorylated at Ser10 and Thr6 are generated by AurB.

We then reacted both mixtures with Chk1 and $\left.{ }^{32} \mathrm{P}\right] \mathrm{ATP}$. Although we readily detected ${ }^{32} \mathrm{P}$ incorporation into the non-AurB-modified nucleosomal control sample, we did not observe any phosphorylation on pSer10 and phospho-Ser28 (pSer28) nucleosomes (Fig. 2b). Notably, phosphorylation-mimicking mutations such as S10D and S10E only partially reflected the Chk1 inhibitory potential of phosphorylated Ser10 (Supplementary Fig. 1d).

Next, we asked whether phosphorylation of Thr11 also affected phosphorylation of Ser10 in a reciprocal manner. To this end, we probed the mixture of Chk1 pSer10- and pThr11-modified peptides with AurB or Msk1 (Fig. 2c and Supplementary Fig. 1e). In both instances, Ser10 and, eventually, Ser28 were phosphorylated indistinguishably from unmodified substrates. In contrast to Chk1, the activities of AurB and Msk1 were not affected by the phosphorylation status of Thr11. In addition, neither kinase alone produced mutually exclusive modification patterns on individual histone $\mathrm{H} 3$ peptides, but they always phosphorylated Ser10 and Ser 28 on all H3 molecules (Supplementary Fig. If and ref. 15). We confirmed the presence of Ser10-and-Thr11 doubly phosphorylated species by NMR analysis of a synthetic $\mathrm{H} 3$ peptide phosphorylated on both Ser10 and Thr11 (Fig. 2d). Molecular dynamics (MD) simulations of Chk1 and AurB complexes bound to non- or premodified H3 substrates supported the notion that $\mathrm{H} 3 \mathrm{Ser} 10$ phosphorylation was incompatible with Chk1 binding, whereas Thr11 phosphorylation had no effect on the interaction with AurB (Supplementary Fig. 2a,b).

\section{Phosphorylation of $\mathrm{H} 3 \mathrm{Thr} 6$ and Ser10 by PKC $\alpha$}

To investigate whether similar cross-talk also existed for other $\mathrm{H} 3$ phosphorylation reactions, we analyzed Thr6 modification by members of the PKC family of kinases ${ }^{7}$. Although PKC $\alpha$ modified $~ 70 \%$ of all $\mathrm{H} 3$ molecules at Thr6, we also detected phosphorylation of Ser10 ( 30\%) (Fig. 3a). Similar phosphorylation properties were observed for the PKC $\beta I$ and PKC $\beta I I$ kinase isoforms (Supplementary Fig. 3a,b). Time-resolved NMR monitoring of the PKC $\alpha$ reaction revealed a clear preference for Thr6 phosphorylation over Ser10 modification, which was preserved at different substrate concentrations (Fig. 3b,c). In analogy to Chk1, Thr6 and Ser10 were always modified on different $\mathrm{H} 3$ molecules and never modified on the same tail peptide (Fig. 3d). This suggested that modification of Ser10 exerted a similar inhibitory effect on Thr6 phosphorylation by the PKC family of kinases as that previously determined for Thr11 phosphorylation by Chk1. To test whether premodification of Ser 10 was also mutually exclusive with and inhibitory to Thr6 phosphorylation, we prephosphorylated the histone $\mathrm{H} 3$ tail peptide at both Ser 10 and Ser 28 and then reacted the product with PKC $\alpha$. No modification of Thr6 was detected (Supplementary Fig. 3c). In contrast, prephosphorylated Thr6 did not affect the subsequent modifications of Ser10 and Ser28 by AurB or Msk1 (Fig. 3e and data not shown). As with Chk1 activity at Thr11, H3 mutations mimicking Ser10 phosphorylation had little effect on PKC $\alpha$-mediated modification of Thr6 (Supplementary Fig. 3d). These results indicated that modification of Ser 10 exerted a similar inhibitory effect on Thr6 phosphorylation by the PKC family of kinases as on Thr11 phosphorylation by Chk1.

\section{H3 Thr11 phosphorylation impedes Lys14 acetylation}

Phosphorylation of Ser10, as well as that of Thr11, has been suggested to promote gene expression by enhancing acetylation of neighboring 
a
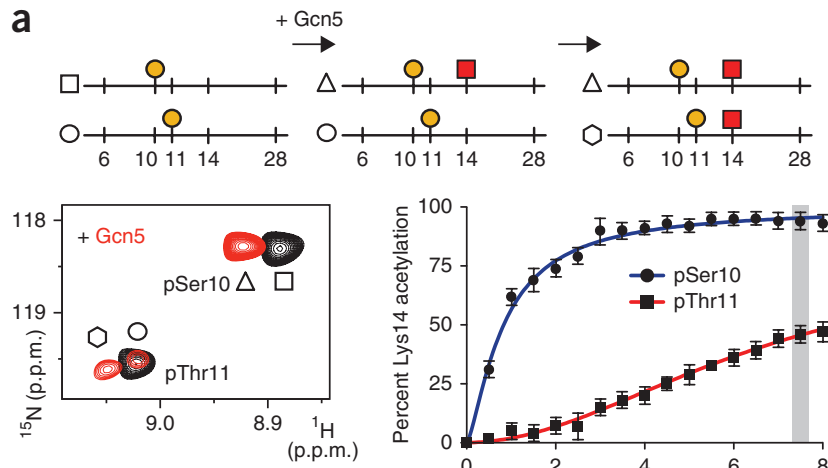

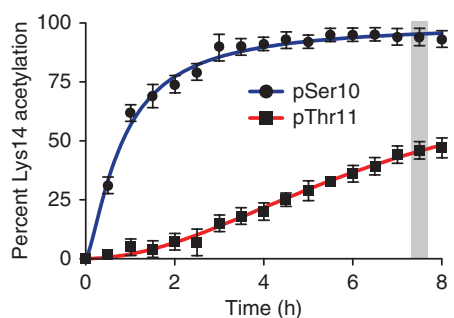

Figure 4 H3 Thr11 phosphorylation impedes Lys14 acetylation by Gcn5. (a) Intermolecular competition reaction of a mixture of ${ }^{15} \mathrm{~N}$ isotope-labeled pSer10 and pThr11 H3 substrates with Gcn5. Close-up view of $2 \mathrm{D}^{1} \mathrm{H}-{ }^{15} \mathrm{~N}$ NMR spectra (overlay; left) of Gcn5 Lys 14 acetylation-induced chemicalshift changes of pSer10 and pThr11 resonance cross-peaks (red).

Time-resolved Lys 14 acetylation profiles of the mixture of H3 pSer10 and pThr11 peptides (right; two independent experiments). Error bars represent s.d.

(b) Chk1-mediated Ser10 and Thr11 phosphorylation of Lys14-preacetylated H3. (c) Intramolecular reaction hierarchies determined in this study. P, phosphorylated; Ac, acetylated. (d) Schematic depictions of hypothetical sets of asymmetric histone H3 modification marks on individual nucleosomes. Yellow circle, phosphorylation; red square, acetylation.

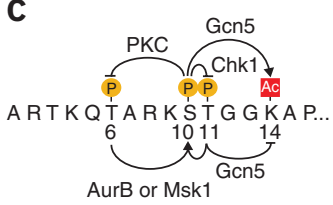

b
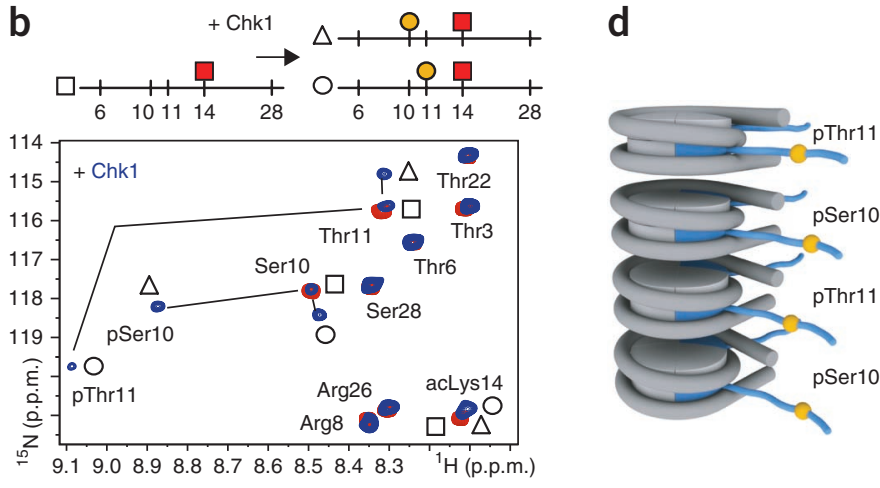

C

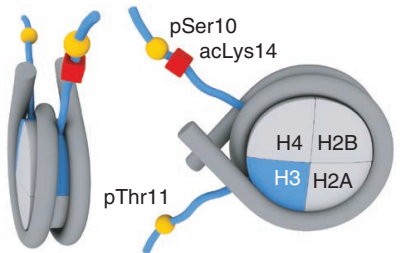

Lys9 (ref. 11) and Lys14 (ref. 16,17) by the prototypical histone acetyltransferase (HAT) Gcn5. However, other studies using full-length Gcn5 in the context of the Spt-Ada-Gcn5 acetyltransferase (SAGA) chromatin-remodeling complex questioned these findings ${ }^{18,19}$. We employed recombinant full-length Gcn5 to probe the influence of Ser10 and Thr11 phosphorylation on acetylation of Lys9 and Lys14 in the mixture of $\mathrm{H} 3$ peptides phosphorylated by Chk1. As Ser10- and Thr11-phosphorylated substrate molecules gave rise to discrete sets of NMR signals, we were able to simultaneously follow the respective acetylation reactions in an intermolecular competitive setting. Time-resolved NMR experiments revealed that Lys14 acetylation of Ser10-phosphorylated peptides was accomplished rapidly (Fig. 4a). Once the reaction mixture was depleted of pSer10 molecules, Lys14 acetylation of Thr11-modified peptides was observed, although at a much slower rate. Reduced Lys14 acetylation of Thr11phosphorylated species is in agreement with previous results on the structural requirements of $\mathrm{H} 3$ binding by the acetyltransferase domain of Gcn5 (ref. 20). We also found that Lys14 acetylation of unmodified or Ser10-phosphorylated histone-H3 peptides, reacted under isolated conditions, was accomplished with similar rates ${ }^{18,19}$ (Supplementary Fig. 4a). Within the time frame of the NMR experiment, no Lys9 acetylation was detected on either substrate type. We further determined that preacetylation of Lys14 had no effect on the phosphorylation behaviors of Chk1 (Fig. 4b), AurB and Msk1 (Supplementary Fig. 4b,c).

\section{DISCUSSION}

Our data indicate that two histone H3-modifying kinases, Chk1 and PKC, display dual substrate site specificities that involve Ser 10 phosphorylation as a second mutually exclusive modification event. In this way, both kinases establish nonidentical, histone $\mathrm{H} 3$ copy-specific phosphorylation marks that also impose a mechanistic hierarchy for further modification events (Fig. 4c). Ser10 phosphorylation is detected both in interphase (that is, during transcriptional activation) and, more globally, during mitosis (that is, during chromosome condensation and segregation) ${ }^{21}$, whereas phosphorylation of Thr11 by Chk1, or Thr6 by PKC, has been implicated in transcriptional control in interphase $e^{7,11}$. Similar effects of interphase-specific Ser10 phosphorylation marks and transcription activation have been reported for Msk1 and Msk2 in response to stress-inducing stimuli such as the phorbol ester 12-O-tetradecanoylphorbol-13-acetate (TPA) treatment ${ }^{22}$, as well as for the tumor necrosis factor- $\alpha$-induced kinase IKK- $\alpha^{23}$ and for PIM1 (ref. 24), PKB (Akt) and Rsk2 (ref. 25). Therefore, dual phosphorylation on separate $\mathrm{H} 3$ molecules, at Thr11 and Ser 10 or at Thr6 and Ser10, may constitute gene-specific chromatin signals in interphase that regulate transcriptional activation. Dual Thr11-Ser10 or Thr6-Ser10 phosphorylation may additionally function in fine-tuning the modification states of neighboring lysine residues. Thr11 phosphorylation has been reported to enhance the activity of Jumonji Domain (JMJD) 2C to remove tri-, di- and monomethyl groups from Lys9 (refs. 9,26). In contrast, phosphorylation of Ser10 blocks demethylation of Lys9 by JMJD2A ${ }^{27}$ and, similarly, phosphorylation of Thr6 inhibits demethylation of Lys 4 by lysine-specific histone demethylase (LSD) 1 (ref. 7). We are currently investigating how these lysine methylation marks affect the dual phosphorylation behavior of Chk1 and $\mathrm{PKC}^{28}$. Dual modification patterns may also facilitate multivalent binding of effector molecules to chromatin ${ }^{29}$, which could occur within the same, or neighboring, nucleosomes ${ }^{30}$ (Fig. 4d). Proteins from the 14-3-3 family are prime candidates for such hypothetical interactions because their ability to form homo- or heterodimers may allow them to simultaneously bind phosphorylated Ser10 and Thr11, or Ser10 and Thr6, on different histone H3 tails within the same nucleosome, or to bridge adjacent nucleosomes in a site-specific manner ${ }^{31}$.

We show that Ser10 phosphorylation by AurB is independent of the phosphorylation states of Thr11 and Thr6 on the same $\mathrm{H} 3$ tail. At the onset of mitosis, this may ensure global Ser10 modification, which is necessary for proper chromosome condensation and segregation. By contrast, the activities of Chk1 and PKC are strongly dependent on the phosphorylation status of Ser10, which may prevent de novo modification of Thr11 and Thr6 during mitosis, at times when the bulk of these predominantly cytoplasmic kinases are mixed with nuclear substrates 
after nuclear-envelope breakdown. In that sense, phosphorylation of Ser10 may additionally function as a regulatory master switch that prevents aberrant modifications of other $\mathrm{H} 3$ phosphorylation sites during mitosis. Last, we point out that, in the absence of other factors, Chk1 phosphorylates Ser10 and Thr11 of histone H3 in roughly equal proportions. Hypothetically, this may result in asymmetric phosphorylation marks on the two nucleosomal copies of histone H3 (Fig. 4d). Whether asymmetrically modified nucleosomes occur in a chromosomal context remains an open question, and further work is needed to determine the physiological roles of dual Ser10-Thr6 or Ser10-Thr11 phosphorylation in native chromatin.

\section{METHODS}

Methods and any associated references are available in the online version of the paper.

Note: Supplementary information is available in the online version of the paper.

\section{ACKNOWLEDGMENTS}

We thank P. Schmieder and M. Beerbaum for excellent NMR infrastructure maintenance. A.S. and W.F. acknowledge funding by the Max Planck Gesellschaft (MBG). S.J.E. was supported by a Boehringer Ingelheim Fund (BIF) fellowship. F.-X.T. acknowledges support from the Association pour la Recherche contre le Cancer (ARC). D.S. and P.S. are supported by Emmy Noether research grants (SCHW1163/31 and SE1794/1-1) from the Deutsche Forschungsgemeinschaft (DFG).

\section{AUTHOR CONTRIBUTIONS}

S.L. conceived and performed NMR and biochemical experiments, analyzed the data and wrote the paper. A.S. and S.J.E. conceived and performed biochemical experiments. F.-X.T. performed computational modeling. R.K. performed peptide synthesis. B.v.R. performed structural modeling and visualization. D.S., C.D.A. and W.F. conceived biochemical experiments, discussed data and wrote parts of the manuscript. P.S. developed the project, supervised all experiments, analyzed the data, wrote the manuscript and made the figures. All authors discussed ideas and concepts, carefully read the manuscript and approved of the conclusions drawn therein.

\section{COMPETING FINANCIAL INTERESTS}

The authors declare no competing financial interests.

Published online at http://www.nature.com/doifinder/10.1038/nsmb.2310.

Reprints and permissions information is available online at http://www.nature.com/ reprints/index.html.

1. Banerjee, T. \& Chakravarti, D. A peek into the complex realm of histone phosphorylation. Mol. Cell. Biol. 31, 4858-4873 (2011).

2. Baek, S.H. When signaling kinases meet histones and histone modifiers in the nucleus. Mol. Cell 42, 274-284 (2011)

3. Jenuwein, T. \& Allis, C.D. Translating the histone code. Science 293, 1074-1080 (2001).

4. Latham, J.A. \& Dent, S.Y. Cross-regulation of histone modifications. Nat. Struct. Mol. Biol. 14, 1017-1024 (2007).

5. Davie, J.R. MSK1 and MSK2 mediate mitogen- and stress-induced phosphorylation of histone H3: a controversy resolved. Sci. STKE 2003, PE33 (2003).

6. Wei, Y., Yu, L., Bowen, J., Gorovsky, M.A. \& Allis, C.D. Phosphorylation of histone H3 is required for proper chromosome condensation and segregation. Cell 97, 99-109 (1999).
7. Metzger, E. et al. Phosphorylation of histone $\mathrm{H} 3 \mathrm{~T} 6$ by $\mathrm{PKC}(\mathrm{I}(\mathrm{I})$ controls demethylation at histone H3K4. Nature 464, 792-796 (2010).

8. Garske, A.L. et al. Combinatorial profiling of chromatin binding modules reveals multisite discrimination. Nat. Chem. Biol. 6, 283-290 (2010).

9. Metzger, E. et al. Phosphorylation of histone $\mathrm{H} 3$ at threonine 11 establishes a novel chromatin mark for transcriptional regulation. Nat. Cell Biol. 10, 53-60 (2008).

10. Preuss, U., Landsberg, G. \& Scheidtmann, K.H. Novel mitosis-specific phosphorylation of histone $\mathrm{H} 3$ at Thr11 mediated by DIk/ZIP kinase. Nucleic Acids Res. 31, 878-885 (2003).

11. Shimada, M. et al. Chk1 is a histone $\mathrm{H} 3$ threonine 11 kinase that regulates DNA damage-induced transcriptional repression. Cell 132, 221-232 (2008).

12. Selenko, P. et al. In situ observation of protein phosphorylation by high-resolution NMR spectroscopy. Nat. Struct. Mol. Biol. 15, 321-329 (2008).

13. Hsu, J.Y. et al. Mitotic phosphorylation of histone H3 is governed by Ipl1/aurora kinase and Glc7/PP1 phosphatase in budding yeast and nematodes. Cell 102 279-291 (2000)

14. Lau, P.N. \& Cheung, P. Histone code pathway involving H3 S28 phosphorylation and $\mathrm{K} 27$ acetylation activates transcription and antagonizes polycomb silencing. Proc. Natl. Acad. Sci. USA 108, 2801-2806 (2011).

15. Liokatis, S., Dose, A., Schwarzer, D. \& Selenko, P. Simultaneous detection of protein phosphorylation and acetylation by high-resolution NMR spectroscopy. J. Am. Chem. Soc. 132, 14704-14705 (2010).

16. Cheung, P. et al. Synergistic coupling of histone H3 phosphorylation and acetylation in response to epidermal growth factor stimulation. Mol. Cell 5, 905-915 (2000).

17. Lo, W.S. et al. Phosphorylation of serine 10 in histone $\mathrm{H} 3$ is functionally linked in vitro and in vivo to Gen5-mediated acetylation at lysine 14. Mol. Cel/ 5, 917-926 (2000).

18. Fry, C.J., Shogren-Knaak, M.A. \& Peterson, C.L. Histone H3 amino-terminal tail phosphorylation and acetylation: synergistic or independent transcriptional regulatory marks? Cold Spring Harb. Symp. Quant. Biol. 69, 219-226 (2004).

19. Shogren-Knaak, M.A., Fry, C.J. \& Peterson, C.L. A native peptide ligation strategy for deciphering nucleosomal histone modifications. J. Biol. Chem. 278, 15744-15748 (2003).

20. Clements, A. et al. Structural basis for histone and phosphohistone binding by the GCN5 histone acetyltransferase. Mol. Cell 12, 461-473 (2003).

21. Nowak, S.J. \& Corces, V.G. Phosphorylation of histone H3: a balancing act between chromosome condensation and transcriptional activation. Trends Genet. 20 214-220 (2004).

22. Soloaga, A. et al. MSK2 and MSK1 mediate the mitogen- and stress-induced phosphorylation of histone H3 and HMG-14. EMBO J. 22, 2788-2797 (2003).

23. Yamamoto, Y., Verma, U.N., Prajapati, S., Kwak, Y.T. \& Gaynor, R.B. Histone H3 phosphorylation by IKK- $\alpha$ is critical for cytokine-induced gene expression. Nature 423, 655-659 (2003).

24. Zippo, A., De Robertis, A., Serafini, R. \& Oliviero, S. PIM1-dependent phosphorylation of histone $\mathrm{H} 3$ at serine 10 is required for MYC-dependent transcriptional activation and oncogenic transformation. Nat. Cell Biol. 9, 932-944 (2007).

25. Sassone-Corsi, P. et al. Requirement of Rsk-2 for epidermal growth factor-activated phosphorylation of histone H3. Science 285, 886-891 (1999).

26. Wissmann, M. et al. Cooperative demethylation by JMJD2C and LSD1 promotes androgen receptor-dependent gene expression. Nat. Cell Biol. 9, 347-353 (2007).

27. Ng, S.S. et al. Crystal structures of histone demethylase JMJD2A reveal basis for substrate specificity. Nature 448, 87-91 (2007).

28. Theillet, F.X. et al. Site-specific mapping and time-resolved monitoring of Lysine methylation by high-resolution NMR spectroscopy. J. Am. Chem. Soc. 134, 7616-7619 (2012).

29. Ruthenburg, A.J., Li, H., Patel, D.J. \& Allis, C.D. Multivalent engagement of chromatin modifications by linked binding modules. Nat. Rev. Mol. Cell Biol. 8 , 983-994 (2007).

30. Ruthenburg, A.J. et al. Recognition of a mononucleosomal histone modification pattern by BPTF via multivalent interactions. Cell 145, 692-706 (2011).

31. Taverna, S.D., Li, H., Ruthenburg, A.J., Allis, C.D. \& Patel, D.J. How chromatinbinding modules interpret histone modifications: lessons from professional pocket pickers. Nat. Struct. Mol. Biol. 14, 1025-1040 (2007). 


\section{ONLINE METHODS}

Expression, uniform isotope labeling and purification of recombinant histone H3. Uniformly ${ }^{15} \mathrm{~N}$ isotope-labeled recombinant wild-type human histone $\mathrm{H} 3$ (residues 1-33) was produced as previously described ${ }^{32}$. The purified products contain an additional glycine residue at the $\mathrm{N}$ termini that results from the TEV cleavage reaction.

Nucleosome reconstitution. Mononucleosomes were reconstituted as previously described $^{33}$, using the 601 DNA sequence ${ }^{34}$ and recombinant Xenopus laevis core histones. The 147-base-pair 601 sequence was generated by PCR amplification using the following primers: 601-forward 5'-TGGAGAATCCCGGTGCCGAGGC-3' and 601-reverse 5'-ACAGGATGTATATATCTGACACGTGCCTG-3'. The PCR product was purified by phenol-chloroform extraction and precipitated with isopropanol. The integrity and monodispersity of the nucleosome preparations were assessed by $6 \%$ native PAGE DNA electrophoresis and analytical ultracentrifugation.

Generation of synthetic $\mathrm{H} 3$ peptides (site-specific phosphorylated and nonphosphorylated). Human $\mathrm{H} 3$ peptides were synthesized using standard Fmocbased solid-phase chemistry on an Intavis Respep XL synthesizer. Further details can be found in the Supplementary Note.

Recombinant enzymes. Aurora B (AurB) and Chk1 kinases were purchased from SignalChem. PKC isoforms were purchased from ProQinase. MSK1 kinase was purchased from Millipore. Full-length Gen 5 from Saccharomyces cerevisiae was expressed and purified from non-isotope-labeled LB cultures, as a histidinetagged fusion protein, using standard Ni-NTA purification and size-exclusion chromatography (Hi-load Superdex 75, GE Healthcare).

In vitro phosphorylation and acetylation reactions (NMR readout). Uniformly ${ }^{15} \mathrm{~N}$-labeled histone $\mathrm{H} 3$ substrates were assayed at final NMR concentrations of $20 \mu \mathrm{M}$ in $20 \mathrm{mM} \mathrm{NaPO}_{4}, 25 \mathrm{mM} \mathrm{NaCl}, 1 \mathrm{mM}$ DTT, $5 \mathrm{mM} \mathrm{MgCl}_{2}, 1 \mathrm{mM}$ PMSF, at $\mathrm{pH}$ 6.9. Phosphorylation reactions were performed with $0.5 \mathrm{mM}$ ATP and $0.5 \mu \mathrm{g}$ of each kinase (final concentrations: $18 \mathrm{nM}, 22 \mathrm{nM}, 16 \mathrm{nM}, 14 \mathrm{nM}$ for Chk1, AurB, Msk1 and PKC isoforms, respectively). Reactions were allowed to proceed for $12 \mathrm{~h}$ at $303 \mathrm{~K}$, except for the complete AurB kinetic-profiling scheme in Supplementary Figure 1f, which was performed at $295 \mathrm{~K}$. Here, time-resolved reaction profiles were fitted using allosteric sigmoidal models in GraphPad Prism 5.0. Acetylation was obtained by addition of $0.5 \mathrm{mM}$ acetyl-CoA and recombinant $\mathrm{Gcn} 5$ at a final concentration of $1 \mu \mathrm{M}$. Reactions were allowed to proceed for $3 \mathrm{~h}$ at $303 \mathrm{~K}$ or at $295 \mathrm{~K}$ for kinetic profiling. Whenever two different enzymes were used in a consecutive fashion, the first reaction was stopped at the indicated time points by heat inactivating the enzyme at $65^{\circ} \mathrm{C}$ for $30 \mathrm{~min}$. The reaction mixture was then cooled to $303 \mathrm{~K}$, and the second enzyme and cofactors were added. Control experiments indicated that the primary enzymes were efficiently heat inactivated by this procedure (data not shown). Kinetic profiles were fitted to first-order rate equations. For the acetylation curves of pSer10- and pThr11premodified species in Figure 4a, single-exponential buildups and allosteric sigmoidal models were used, respectively, in GraphPad Prism 5.0.

Phosphorylation assays and western blotting. For phosphorylation reactions and western-blot analyses, $0.6 \mu \mathrm{g}$ of $\mathrm{H} 3$ (1-33) and $1 \mu \mathrm{g}$ of full-length $\mathrm{H} 3$ or nucleosomes were mixed in kinase buffer $(10 \mathrm{mM}$ Tris- $\mathrm{HCl}, \mathrm{pH} 7.5,5 \mathrm{mM}$ $\mathrm{MgCl}_{2}, 20 \mathrm{mM} \mathrm{NaCl}, 1 \mathrm{mM}$ EDTA, $2 \mathrm{mM} \mathrm{DTT}$ ) in the presence of $0.5 \mathrm{mM}$ ATP. Phosphorylation reactions were incubated at $303 \mathrm{~K}$ for $12 \mathrm{~h}$ in the presence of $100 \mathrm{ng}$ Chk1. In the case of nucleosomes, additional reactions in the presence of $200 \mathrm{ng}$ and $300 \mathrm{ng}$ of Chk1 were performed. Samples were run on 18\% SDS-PAGE gels and transferred onto PVDF membranes. Western blots were developed in TBS-T with $5 \%$ nonfat dry milk powder using the following antibodies: anti-H3 (H9289, Sigma; 1:1,000 dilution), anti-H3pSer10 (06-570, Millipore; 1:5,000 dilution), anti-H3pThr11 (05-789, Millipore, 1:1,000) and anti-rabbit IgG (A6667, Sigma; 1:5,000 dilution).

$\left[{ }^{32} \mathrm{P}\right] \mathrm{ATP}$ radionucleotide incorporation assays. Kinase reactions were performed using $1 \mu \mathrm{g}$ nucleosomes, $5 \mathrm{ng}$ Chk $1,50 \mu \mathrm{M}$ ATP and $0.03 \mathrm{mCi} / \mathrm{ml}$ $\left[\gamma_{-}{ }^{32} \mathrm{P}\right]$ ATP in kinase buffer ( $5 \mathrm{mM}$ MOPS, $\mathrm{pH} 7.2,5 \mathrm{mM} \mathrm{MgCl}, 1 \mathrm{mM}$ EGTA, $0.4 \mathrm{mM}$ EDTA, $0.05 \mathrm{mM}$ DTT, $2.5 \mathrm{mM} \beta$-glycerophosphate). Reactions were stopped after $1 \mathrm{~h}$ at $30^{\circ} \mathrm{C}$ by adding SDS sample buffer, and were analyzed by autoradiography following SDS-PAGE. In the case of histone peptides (H3 residues 1-15), $5 \mu \mathrm{g}$ peptide and $100 \mathrm{ng}$ Chk1 were used. Radiolabeled peptides were spotted on P81 paper, washed three times with $75 \mathrm{mM}$ phosphoric acid and once with acetone and analyzed by scintillation counting. Phosphorylation of nucleosomes by AurB was performed as described above, using $10 \mathrm{ng}$ AurB per $\mu \mathrm{g}$ of nucleosome and omitting $\left[\gamma^{-32} \mathrm{P}\right] \mathrm{ATP}$. The reaction was stopped by heat inactivation for $30 \mathrm{~min}$ at $65^{\circ} \mathrm{C}$. Integrity of nucleosomes after heat shock was assessed by $6 \%$ native PAGE DNA electrophoresis.

NMR spectroscopy. All NMR spectra were recorded on a 750-MHz Bruker Avance spectrometer equipped with cryogenically cooled triple-resonance ${ }^{1} \mathrm{H}\left({ }^{13} \mathrm{C} /{ }^{15} \mathrm{~N}\right)$ probe $(\mathrm{TCI})$. For natural-abundance NMR measurements of nonisotope-labeled synthetic $\mathrm{H} 3$ peptides (unmodified and site-selectively phosphorylated), lyophilized samples were dissolved in $20 \mathrm{mM} \mathrm{NaPO}_{4}, 25 \mathrm{mM} \mathrm{NaCl}, \mathrm{pH}$ 6.9 , to a final concentration of $2 \mathrm{mM} .2 \mathrm{D}^{1} \mathrm{H}^{-15} \mathrm{~N}$ SOFAST-HMQC NMR spectra were recorded at $282 \mathrm{~K}$ with $1 \mathrm{~K}$ transients and $1,024\left({ }^{1} \mathrm{H}\right) \times 256\left({ }^{15} \mathrm{~N}\right)$ complex points (acquisition time $\sim 11 \mathrm{~h}$ ). In vitro phosphorylation reactions of recombinant $\mathrm{H} 3$ substrates were measured by $2 \mathrm{D}^{1} \mathrm{H}^{15} \mathrm{~N}$ SOFAST-HMQC experiments at $282 \mathrm{~K}$ with 64 transients and $1,024\left({ }^{1} \mathrm{H}\right) \times 128\left({ }^{15} \mathrm{~N}\right)$ complex points (acquisition time $\sim 18 \mathrm{~min}$ ). The lower temperature for measuring the NMR experiments was chosen because of unfavorable chemical-exchange properties of serine residues in their nonphosphorylated forms at $303 \mathrm{~K}$. Average phosphorylation levels were calculated from respective peak intensities (modified/unmodified). All NMR spectra were processed with iNMR 3.3.9 without apodization functions. NMR assignments of resonance frequencies of phosphorylated amino acids, as well as of neighboring substrate sites experiencing chemical shift changes, were confirmed by heteronuclear triple-resonance NMR experiments (HNCO, HNCA, HNCAB) on ${ }^{15} \mathrm{~N} /{ }^{13} \mathrm{C}$-labeled $\mathrm{H} 3$ substrates (as also described in ref. 32 ).

Kinase substrate models. Models were obtained from a simulated annealing procedure using AMBER9 (ref. 35). Crystal structures of Chk1 (ref. 36), Aurora $\mathrm{B}^{37}$ and $\mathrm{PHK} \gamma \mathrm{t}^{38}$ were used to build initial coordinate files. Further details are available in the Supplementary Note.

32. Liokatis, S., Dose, A., Schwarzer, D. \& Selenko, P. Simultaneous detection of protein phosphorylation and acetylation by high-resolution NMR spectroscopy. J. Am. Chem. Soc. 132, 14704-14705 (2010).

33. Luger, K., Rechsteiner, T.J. \& Richmond, T.J. Expression and purification of recombinant histones and nucleosome reconstitution. Methods Mol. Biol. 119, 1-16 (1999).

34. Lowary, P.T. \& Widom, J. New DNA sequence rules for high affinity binding to histone octamer and sequence-directed nucleosome positioning. J. Mol. Biol. 276, 19-42 (1998).

35. Case, D.A. et al. The Amber biomolecular simulation programs. J. Comput. Chem. 26, 1668-1688 (2005).

36. Chen, P. et al. The $1.7 \AA$ crystal structure of human cell cycle checkpoint kinase Chk1: implications for Chk1 regulation. Cell 100, 681-692 (2000).

37. Sessa, F. et al. Mechanism of Aurora B activation by INCENP and inhibition by hesperadin. Mol. Cell 18, 379-391 (2005).

38. Lowe, E.D. et al. The crystal structure of a phosphorylase kinase peptide substrate complex: kinase substrate recognition. EMBO J. 16, 6646-6658 (1997). 